\title{
Image charges in semiconductor quantum wells: Effect on exciton binding energy
}

\author{
D. B. Tran Thoai* \\ Fritz-Haber-Institut der Max-Planck-Gesellschaft zur Fönderung der Wissenschaften, \\ Faradayweg 4-6, D-1000 Berlin 33, Federal Republic of Germany \\ R. Zimmermann \\ Zentralinstitut für Elektronenphysik der Akademie der Wissenschaften der Deutschen Demokratischen Republik, \\ Hausvogteiplatz 5-7, DDR-1086 Berlin, German Democratic Republic
}

M. Grundmann and D. Bimberg

Institut für Festkörperphysik I, Technische Universität Berlin, Hardenbergstrasse 36, D-1000 Berlin 12, Federal Republic of Germany

(Received 29 May 1990)

\begin{abstract}
Binding energies of excitons in a quantum-well structure are calculated including fully the effects of image charges, finite barriers, the $z$ correlation of electrons and holes, and anisotropic hole masses. The influence of discontinuous masses and discontinuous dielectric constants across the interfaces is evaluated in detail: While the mass difference becomes important only when the excitonic wave function penetrates into the barrier, the image charges appreciably modify the Coulomb interaction and therefore influence the exciton binding energy even at well widths larger than the exciton Bohr radius. Results for technologically important, particular material systems are presented.
\end{abstract}

The well-recognized importance of excitonic effects on the optical properties of semiconductor quantum wells (QW's) (Refs. 1-3) has attracted much attention to the calculation of exciton binding energies in these structures. $^{4-13}$ Although the problem is well defined, theoretical calculations for realistic structures with different material parameters in the well and barrier region were carried out with several approximations up to now. In contrast, three-dimensional (3D) excitons in bulk semiconductors have been treated with much greater precision. ${ }^{14,15}$ In the investigations on excitons in QW's by Miller et al. ${ }^{4}$ and Bastard et al.,$^{5}$ an infinite-barrier potential has been used. The theory has been largely improved by Green and Bajaj, ${ }^{6}$ who took finite barriers into account. But, they used the same masses and dielectric constants for the well and barrier material, thus avoiding some theoretical difficulties. Grundmann and Bimberg ${ }^{7}$ presented a calculation which yielded the correct limit for the barrier exciton as the $\mathrm{QW}$ width $L_{z} \rightarrow 0$. The $z$ correlation of electrons and holes-increasingly important as $L_{z} \rightarrow 0$ and $L_{z} \rightarrow \infty-$ was properly taken into account by using a nonseparable two-parameter trial wave function for the variational ansatz.

One important issue has been neglected up to this point: The different dielectric constants in the well $\left(\epsilon_{w}\right)$ and barrier $\left(\epsilon_{b}\right)$ region cause image charges yielding potentially significant corrections to the binding energies. Keldysh ${ }^{8}$ has treated this problem for large ratios of well to barrier dielectric constants and $\epsilon_{b} \ll \epsilon_{w}$, typical for semiconductor-insulator heterostructures. He also used an infinite barrier height. For semiconductor QW's with arbitrary dielectric constants, the image charges have been included into such calculations by Tran Thoai, ${ }^{9}$ still with infinite barriers. Whittaker ${ }^{10}$ has included the effect of image charges into the numerical integration of the radial Schrödinger equation with finite barriers (this approach without image charges has been published by $\mathrm{Wu}^{11}$ also). Whittaker's calculation, however, neglects the image self-energy and does not account for the $z$ correlation of electrons and holes. A calculation of the exciton problem with finite barriers, including the image potential and self-energy and the $z$ correlation of electrons and holes, has been published recently by Kumagai and Takagahara. ${ }^{12}$ However, the well-known and essential mass anisotropy for heavy and light holes in the QW is not taken into consideration. ${ }^{12}$

In this paper we calculate the exciton binding energy in a QW with finite barriers, with full inclusion of effects due to the image charges and the $z$ correlation of electrons and holes and with discontinuous masses (taking into account the mass anisotropy of heavy and light holes). The image self-energy is investigated with emphasis on a proper renormalization of the inherent divergence, thus refining the approach adopted in Ref. 12. Therefore, we present a solution of the exciton problem in a quantum well without approximations, except for the valence-band hybridization which might have a certain influence on excitonic spectra. ${ }^{13}$ We use the two-parameter variational function given in Ref. 7 and thus obtain the correct bulk limits as $L_{z} \rightarrow 0$ and as $L_{z} \rightarrow \infty$. The impact of discontinuous masses and/or discontinuous dielectric constants on the exciton binding energy is evaluated. With our ansatz we calculate binding energies for the material combinations $\mathrm{Al}_{0.40} \mathrm{Ga}_{0.60} \mathrm{As} / \mathrm{GaAs}, \mathrm{In}_{0.53} \mathrm{Ga}_{0.47} \mathrm{As} / \mathrm{InP}$, and $\mathrm{In}_{0.53} \mathrm{Ga}_{0.47^{-}}$ $\mathrm{As} / \mathrm{In}_{0.52} \mathrm{Al}_{0.48} \mathrm{As}$, which are presently some of the most important systems for device applications. The material parameters used are listed in Table I.

We now give a brief summary of the theory. The Ham- 
TABLE I. Material parameters of $\mathrm{GaAs}, \mathrm{Al}_{0.40} \mathrm{Ga}_{0.60} \mathrm{As}$, InP, $\mathrm{In}_{0.53} \mathrm{Ga}_{0}{ }_{47} \mathrm{As}$, and $\mathrm{In}_{0.52} \mathrm{Al}_{0.48} \mathrm{As}$ used in the calculation ( $4 \mathrm{~K}$ values).

\begin{tabular}{|c|c|c|c|c|c|c|}
\hline Material & $E_{\text {gap }}(\mathrm{eV})$ & $\Delta E_{c} / \Delta E_{g}$ & $\gamma_{1}$ & $\gamma_{2}$ & $m_{e}$ & $\epsilon$ \\
\hline $\mathrm{GaAs}^{\mathrm{a}}$ & 1.518 & \multirow{2}{*}{$60 \%{ }^{e}$} & 6.85 & 2.1 & 0.0665 & 12.5 \\
\hline $\mathrm{Al}_{0.40} \mathrm{Ga}_{0.60} \mathrm{As}^{\mathrm{b}}$ & 2.163 & & 4.67 & 1.17 & 0.0895 & 11.5 \\
\hline $\operatorname{In} P^{a}$ & 1.423 & \multirow[t]{2}{*}{$40 \%{ }^{f}$} & 5.15 & 0.94 & 0.0803 & 12.6 \\
\hline $\mathrm{In}_{0.53} \mathrm{Ga}_{0.47} \mathrm{As}$ & $0.813^{c}$ & & $11.0^{\mathrm{h}}$ & $4.18^{h}$ & $0.041^{h}$ & $13.9^{\prime}$ \\
\hline $\mathrm{In}_{0.52} \mathrm{Al}_{0.48} \mathrm{As}$ & $1.511^{\mathrm{d}}$ & $75 \%^{g}$ & $4.57^{d}$ & $1.19^{d}$ & $0.074^{d}$ & $12.3^{\mathrm{i}}$ \\
\hline
\end{tabular}

iltonian is given as

$$
\begin{aligned}
H= & -\frac{\hbar^{2}}{2} \frac{\partial}{\partial z_{e}} \frac{1}{m_{e}} \frac{\partial}{\partial z_{e}}-\frac{\hbar^{2}}{2} \frac{\partial}{\partial z_{h}} \frac{1}{m_{h, \perp}} \frac{\partial}{\partial z_{h}} \\
& -\frac{\hbar^{2}}{2 m_{e}} \Delta_{\rho_{e}}-\frac{\hbar^{2}}{2 m_{h, \|}} \Delta_{\rho_{h}}+V_{e, \text { conf }}\left(z_{e}\right)+V_{h, \text { conf }}\left(z_{h}\right) \\
& +V_{e-h}\left(\mathbf{r}_{e}, \mathbf{r}_{h}\right)+\Sigma\left(z_{e}\right)+\Sigma\left(z_{h}\right) .
\end{aligned}
$$

$m_{e}$ is the electron effective mass, $m_{h, \perp}$ and $m_{h, \|}$ are the hole effective masses in $z$ and in-plane directions, respectively, and $\mathbf{r}_{e}=\left(\rho_{e}, z_{e}\right)$. They are expressed in terms of the Luttinger parameters, ${ }^{16} m_{0}$ being the free-electron mass lupper sign is for heavy holes $(\mathrm{HH})$, and lower sign is for light holes $(\mathrm{LH})$ ]:

$$
\frac{1}{m_{h, \|}}=\frac{\gamma_{1} \pm \gamma_{2}}{m_{0}} \text { and } \frac{1}{m_{h, \perp}}=\frac{\gamma_{1} \mp 2 \gamma_{2}}{m_{0}}
$$

$V_{e-h}\left(\mathbf{r}_{e}, \mathbf{r}_{h}\right)$ is the Coulomb potential including the effect of image charges obtained from the solution of the Poisson equation for the QW situation, and $\Sigma(z)$ the corresponding self-energy

$$
\Sigma(z)=\frac{1}{2} \lim _{r^{\prime} \rightarrow r}\left[V_{e-h}\left(r^{\prime}, r\right)-V_{e-h}^{\text {bulk }}\left(r^{\prime}, r\right)\right]
$$

with $V_{e-h}^{\text {bulk }}$ being the bulk Coulomb potential screened with $\epsilon_{w}\left(\epsilon_{b}\right)$ in the well (barrier) region. For the sake of further discussion, we give it explicitly, ${ }^{12}$

$$
\begin{aligned}
& \Sigma^{\text {well }}(z)=\frac{e^{2}}{2 \epsilon_{w}}\left[\sum_{n=1,2, \ldots}^{\infty} \frac{Q^{2 n}}{n L_{z}}+\sum_{n=1,3, \ldots}\left(\frac{Q^{n}}{n L_{z}-2|z|}+\frac{Q^{n}}{n L_{z}+2|z|}\right)\right], \\
& \Sigma^{\text {barrier }}(z)=\frac{e^{2}}{2 \epsilon_{b}} \sum_{n}^{\infty}\left(\frac{Q^{n}}{n L_{z}+2|z|}-\frac{Q^{n}}{(n-2) L_{z}+2|z|}\right) .
\end{aligned}
$$

$Q=\left(\epsilon_{w}-\epsilon_{b}\right) /\left(\epsilon_{w}+\epsilon_{b}\right)$ is a measure of the dielectric misfit. Please note that $\Sigma^{\text {barrier }}$ vanishes for $L_{z}=0$ (no well present). As known, the image self-energy diverges if the boundary is approached, i.e., as $z \rightarrow \pm L_{z} / 2$. This is due to the unphysical assumption that the induced surface charge has no spatial extension. For a metal-semiconductor contact, the divergence is removed by slightly displacing the boundary plane (mirror shift $L_{z} \rightarrow L^{\text {eff }}$ ) into the metal, since the induced density is located there. The same procedure has been adopted in Ref. 12 for the QW situation. But then, $\Sigma^{\text {barrier }}$ remains finite for $L_{z}=0$ and may produce spurious effects. Instead, we assume the surface charge to be located symmetrically with respect to the boundary, and to have an extension $\delta$ of roughly one lattice constant $a_{0}$. Then, the self-energy is calculated with the principal-value replacement

$$
\frac{1}{n L_{z}-2|z|} \rightarrow \frac{n L_{z}-2|z|}{\left(n L_{z}-2|z|\right)^{2}+\delta^{2}}
$$

in every term of (4), and the correct limit for $L_{z}=0$ is preserved. Our including the image self-energy into the sublevel calculation gives an upward shift which is larger for the hole than for the electron since the latter penetrates more easily into the barrier, averaging better over positive and negative portions of $\Sigma(z)$. For the $\mathrm{Al}_{0.40} \mathrm{Ga}_{0.60} \mathrm{As} / \mathrm{GaAs}$ parameters of Fig. 1, maximum sublevel shifts are found at $L_{z}=2 \mathrm{~nm}$, amounting to 1 and 2 $\mathrm{meV}$ for electrons and holes, respectively.

The trial wave function taken from Ref. 7 is

$\phi\left(\rho, z_{e}, z_{h}\right)=\exp \left(-\frac{1}{a} \sqrt{\rho^{2}+\lambda^{2}\left(z_{e}-z_{h}\right)^{2}}\right) u_{e}\left(z_{e}\right) u_{h}\left(z_{h}\right)$,

with confinement wave functions $u_{e}$ and $u_{h}$. In the excitonic part, $a$ is the Bohr diameter, and $\lambda$ accounts for the dimensionality of the exciton and varies between 0 (quasi-2D case) and 1 (3D bulk case for $L_{z} \rightarrow 0$ and $\left.L_{z} \rightarrow \infty\right)$. $a$ and $\lambda$ are fixed by minimizing the expectation value of the Hamiltonian (1).

At this point we would like to discuss an important detail: The hole masses in Eq. (2) only hold for a truly quasi-2D QW situation with an anisotropy between the $z$ and in-plane directions. In the case of bulk semiconductors with zinc blende lattice the exciton binding energy is calculated to a very good approximation with an isotropic and equal hole mass for $\mathrm{HH}$ and $\mathrm{LH}, m_{0} / \gamma_{1}{ }^{15}$ The corrections due to $\gamma_{2}$ are of the order of a few percent only. In the Hamiltonian the hole masses remain strongly 


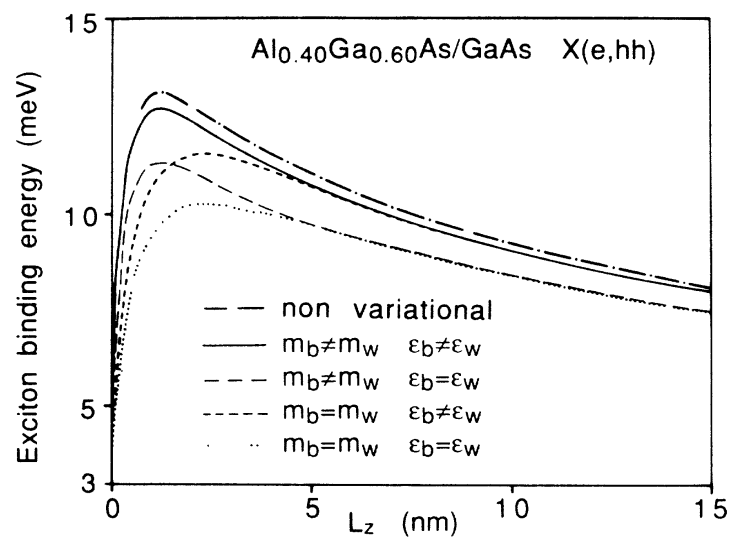

FIG. 1. Heavy-hole exciton binding energy vs quantum well width in $\mathrm{Al}_{0.40} \mathrm{Ga}_{0.60} \mathrm{As} / \mathrm{GaAs} \mathrm{QW}$ 's for different approximations to the barrier parameters. The dash-dotted line is a result from the numerical integration of the $2 \mathrm{D}$ Schrödinger equation.

anisotropic even in the bulk case. As we neglect any $\mathrm{HH}$ LH coupling, we obtain in this limit slightly different wave functions for $\mathrm{HH}$ and $\mathrm{LH} .{ }^{7}$ However, the binding energies come very close to each other.

In all three investigated systems $\mathrm{Al}_{0.40} \mathrm{Ga}_{0.60} \mathrm{As} / \mathrm{GaAs}$, $\mathrm{In}_{0.53} \mathrm{Ga}_{0.47} \mathrm{As} / \mathrm{InP}$, and $\mathrm{In}_{0.53} \mathrm{Ga}_{0.47} \mathrm{As} / \mathrm{In}_{0.52} \mathrm{Al} \mathrm{l}_{0.48} \mathrm{As}$, the barrier material has the larger masses and the smaller dielectric constant. The corrections due to both effects therefore act in the same direction leading to an increase of $E_{b} \sim m / \epsilon^{2}$ as compared to the bulk value of the well material. In Figs. 1 and 2 we have plotted the exciton binding energy as a function of well width for heavy and light holes in an $\mathrm{Al}_{0.40} \mathrm{Ga}_{0.60} \mathrm{As} / \mathrm{GaAs} \mathrm{QW}$ for four different approximations for the ratios of masses and dielectric constants. Taking the barrier mass equal to the well mass and ignoring the image charges (like in Ref. 6), we obtain a rather flat dependence of $E_{b}\left(L_{z}\right)$ which approaches the bulk GaAs value for $L_{z} \rightarrow 0$. The inclusion of image charges does almost not change the shape of the $E_{b}\left(L_{z}\right)$ dependence but leads to a nearly rigid shift of $E_{b}$ even for quite large well widths where the exciton is totally confined in the well region. The image self-energy influences the binding energy only via the modification of the confinement wave function, which is a minor effect.

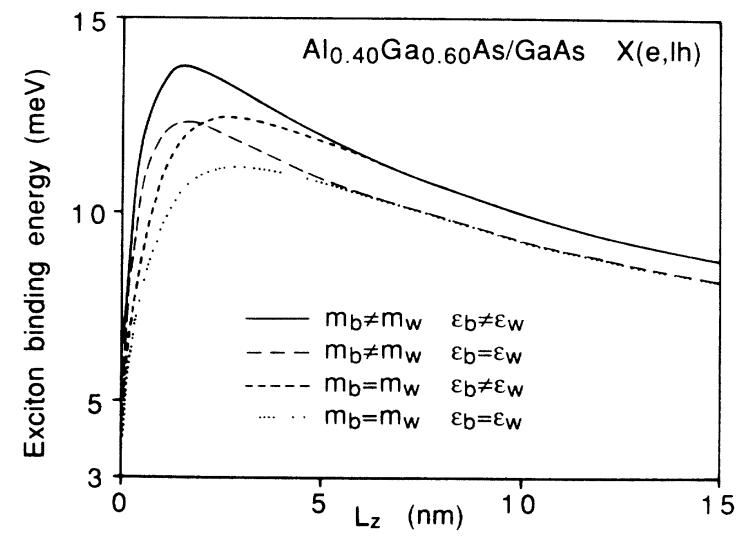

FIG. 2. Light-hole exciton binding energy vs quantum well width in $\mathrm{Al}_{0.40} \mathrm{Ga}_{0.60} \mathrm{As} / \mathrm{GaAs} \mathrm{QW}$ 's for different approximations to the barrier parameters.
The electrostatic interaction manifests itself over larger distances than the exciton Bohr radius $a_{B}$, and the Coulomb interaction in the well is thus substantially modified by the barrier even for $L_{z}>a_{B}$.

Quite different is the influence of the discontinuity of the masses across the interfaces: A change in $E_{b}$ occurs only at well widths where the exciton leaks out into the barrier. For large well width $L_{z}>a_{B}$ the binding energy is not modified. The shape of the $E_{b}$ vs $L_{z}$ dependence changes and gets steeper close to the maximum of the binding energy which shifts to narrower well width. The calculation with the true barrier parameters, including discontinuous masses and image charges, sums up both effects and yields an $E_{b}\left(L_{z}\right)$ curve with a steep maximum at $L_{z} \approx 1.5 \mathrm{~nm}$ and an upward shift at larger well thicknesses. The maximum binding energy fully including the barrier material parameters is about $25 \%$ larger than that obtained from a calculation with the same material parameters for well and barrier. Due to the fact that the $z$ correlation of electrons and holes is fully taken into account we obtain in the limit $L_{z} \rightarrow 0$ for $E_{b}$ the bulk value of the barrier material.

We have included as a dash-dotted line in Fig. 1 the binding energy obtained from a numerical integration of the two-dimensional Schrödinger equation. Here, the $z$ correlation cannot be included and the results are only valid in that range where the variational calculation gave $\lambda$ near to zero ( $1 \mathrm{~nm} \leq L_{z} \leq 20 \mathrm{~nm}$ in the present case). The change in the binding energy is rather small. On the other hand, the variational wave function gives too large values $(\sim 20 \%)$ for the oscillator strength. All further curves in the paper are obtained from the variational calculation.

For $\mathrm{In}_{0.53} \mathrm{Ga}_{0.47} \mathrm{As} / \mathrm{InP}$ (Fig. 3) and $\mathrm{In}_{0.53} \mathrm{Ga}_{0.47} \mathrm{As} /$ $\mathrm{In}_{0.52} \mathrm{Al}_{0.48} \mathrm{As}$ (Fig. 4), we have calculated the heavy- and light-hole binding energy with and without the effect of image charges but in each case considering discontinuous masses. For $\mathrm{In}_{0.53} \mathrm{Ga}_{0.47} \mathrm{As} / \mathrm{InP} \quad\left(\mathrm{In}_{0.53} \mathrm{Ga}_{0.47} \mathrm{As} / \mathrm{In}_{0.52}\right.$ $\mathrm{Al}_{0.48} \mathrm{As}$ ), the binding energy is enlarged by the image charges up to $\sim 15 \%(\sim 20 \%)$.

Our numerical results are partly at variance to those obtained by Kumagai and Takagahara, ${ }^{12}$ who use the same variational function as in this paper [Eq. (6)]. In Fig. 5, we compare their result (dashed line) for the exci-

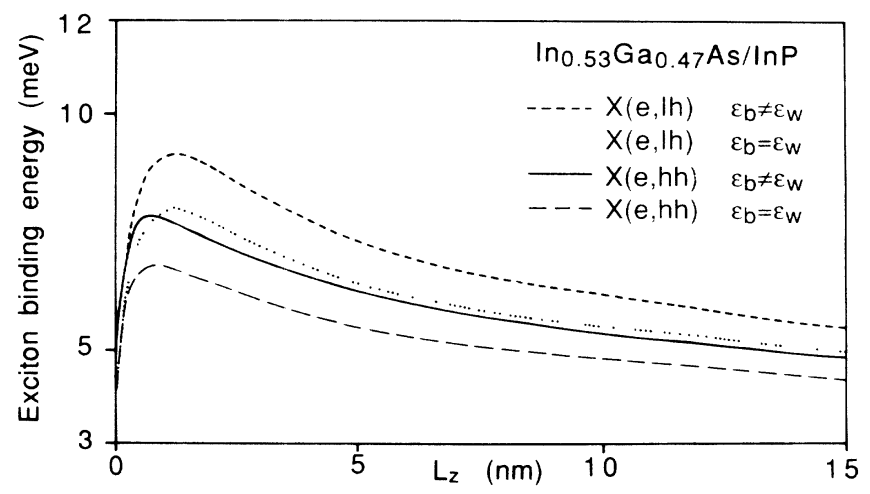

FIG. 3. Exciton binding energy vs quantum well width for heavy and light holes in $\mathrm{In}_{0.53} \mathrm{G}_{0.47} \mathrm{As} / \mathrm{InP} \mathrm{QW}$ 's with and without inclusion of image charges. 


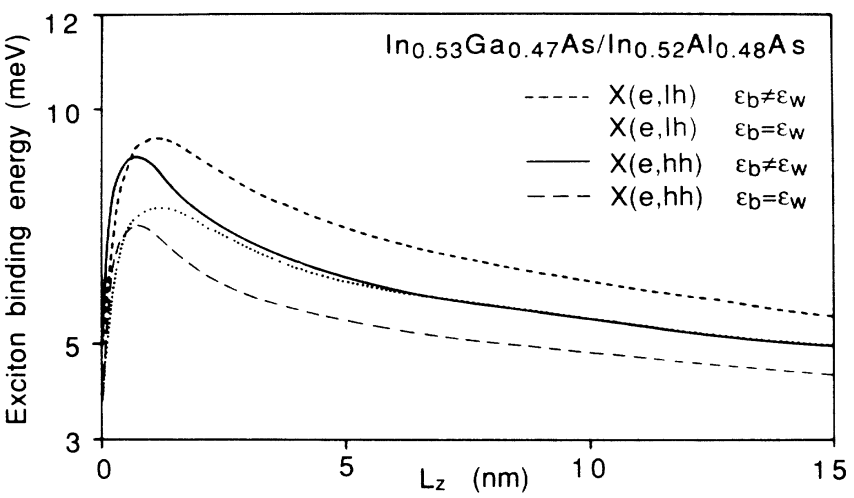

FIG. 4. Exciton binding energy vs quantum well width for heavy and light holes in $\mathrm{In}_{0.53} \mathrm{Ga}_{0.47} \mathrm{As} / \mathrm{In}_{0.52} \mathrm{Al}_{0.48} \mathrm{As} \mathrm{QW}$ 's with and without inclusion of image charges.

ton binding energy in $\mathrm{Al}_{0.30} \mathrm{Ga}_{0.70} \mathrm{As} / \mathrm{GaAs} \mathrm{QW}$ 's with our calculation using the same material parameters. Kumagai and Takagahara do not obtain the drop of binding energy to the barrier bulk value for $L_{z} \rightarrow 0$. This drop is crucial from the theoretical point of view because it is naturally included in the variational ansat $z$ with finite barriers $^{6,7}$ and has been indeed experimentally verified. ${ }^{26,27}$ A possible origin of this discrepancy may be the different treatment of the image self-energy. As detailed above, we have used a scheme which gives zero effect in the limit $L_{z}=0$ as it should, in contrast to Ref. 12 .

Summing up our results, we have evaluated the influence of image charges and discontinuous masses in $\mathrm{QW}$ structures on the exciton binding energy taking the $z$ correlation of electrons and holes into account. The barrier material with the larger band gap usually has the

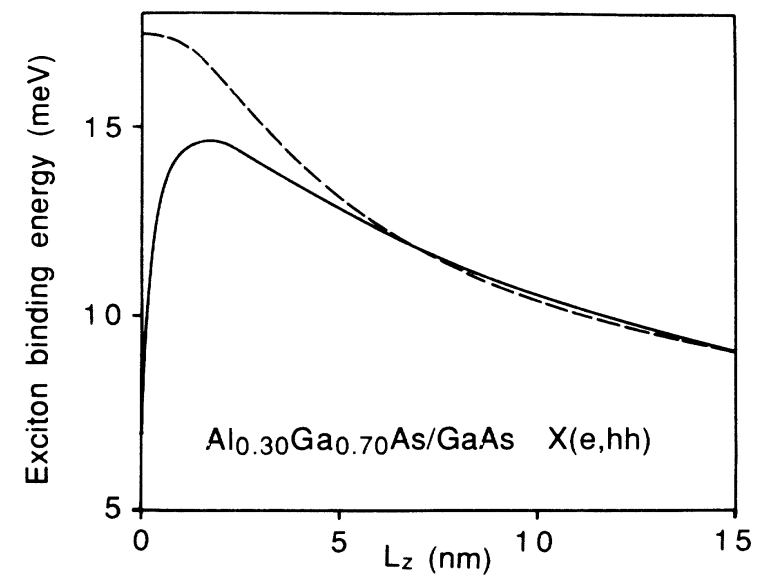

FIG. 5. Heavy-hole exciton binding energy in $\mathrm{Al}_{0.30} \mathrm{Ga}_{0.70} \mathrm{As} /$ GaAs QW's. Solid line, present calculation; dashed line, from Fig. 15 of Ref. 12.

larger masses and the smaller dielectric constant. Both effects increase the binding energy significantly compared to calculations with the same material parameters for the well and barrier region. Our calculation yields the correct bulk binding energies for the limiting cases $L_{z} \rightarrow 0$ and $L_{z} \rightarrow \infty$. The singularity due to the image self-energy has been properly renormalized.

One of the authors (D.B.T.T.) would like to thank Professor Dr. Zeitler for continuous interest and support and the Max-Planck-Gesellschaft for partial support. The work at Technische Universität Berlin is funded by Deutsche Forschungsgemeinschaft (Bonn, Germany), within the framework of Sonderforschungsbereich No. 6.

*Permanent address: National Center for Scientific Research, 1 Mac Dinh Chi, Ho Chi Minh City, Vietnam.

${ }^{1} \mathrm{R}$. Dingle, in Festkörperprobleme: Advances in Solid State Physics, edited by H. J. Queisser (Pergamon, Vieweg, Braunschweig, 1975), Vol. XV, p. 21.

${ }^{2}$ C. Weisbuch, R. C. Miller, R. Dingle, A. C. Gossard, and W. Wiegmann, Solid State Commun. 37, 219 (1981).

${ }^{3}$ D. Chemla and D. A. B. Miller, J. Opt. Soc. Am. B 2, 1155 (1985).

${ }^{4}$ R. C. Miller, D. A. Kleinmann, W. T. Tsang, and A. C. Gossard, Phys. Rev. B 24, 1134 (1981).

${ }^{5}$ G. Bastard, E. E. Mendez, L. L. Chang, and L. Esaki, Phys. Rev. B 26, 1974 (1982).

${ }^{6}$ R. L. Greene and K. K. Bajaj, Solid State Commun. 45, 831 (1983).

${ }^{7}$ M. Grundmann and D. Bimberg, Phys. Rev. B 38, 13486 (1988).

${ }^{8}$ L. V. Keldysh, J. Superlatt. Microstruct. 4, 637 (1988).

${ }^{9}$ D. B. Tran Thoai, Physica B (to be published).

${ }^{10}$ D. M. Whittaker and R. J. Elliott, Solid State Commun. 68, 1 (1988).

"IJ.-W. Wu, Solid State Commun. 69, 1057 (1989).

${ }^{12}$ M. Kumagai and T. Takagahara, Phys. Rev. B 40, 12359 (1989).

${ }^{13}$ B. Zhu and K. Huang, Phys. Rev. B 36, 8102 (1987).

${ }^{14}$ A. Baldereschi and N. O. Lipari, Phys. Rev. Lett. 25, 373 (1970).

${ }^{15}$ A. Baldereschi and N. O. Lipari, Phys. Rev. B 3,439 (1971).

16 J. M. Luttinger, Phys. Rev. 102, 1030 (1956).

${ }^{17}$ Semiconductors, Landolt-Börnstein, New Series, Vol. 17, Pt. a (Springer-Verlag, Berlin, 1982).

${ }^{18}$ For $E_{\text {gap }}$ the bowing parameter $c=0.37$ (Ref. 17) is used; the Luttinger parameters are obtained by linearly interpolation between the masses given in Ref. 17. $\epsilon$ and $m_{e}$ are linear interpolated between the values of GaAs and AlAs given in Ref. 17.

${ }^{19}$ K. H. Goetz, D. Bimberg, H. Juergensen, J. Selders, A. V. Solomonov, G. F. Glinski, and M. Razeghi, J. Appl. Phys. 54, 4543 (1983).

${ }^{20}$ D. Oertel, D. Bimberg, and R. K. Bauer, Appl. Phys. Lett. 55, 140 (1989).

${ }^{21}$ H. Kroemer, Surf. Sci. 174, 299 (1986).

${ }^{22}$ K. Alavi, R. L. Aggarwal, and S. H. Groves, Phys. Rev. B 21, 1311 (1980).

23 J. Böhrer, M. Grundmann, and D. Bimberg (unpublished).

${ }^{24}$ M. S. Skolnick, P. R. Tapster, S. J. Bass, A. D. Pitt, N. Apsley, and S. P. Aldred, Semicond. Sci. Technol. 1, 29 (1986).

${ }^{25}$ Linear interpolation between the values given in Ref. 17 for InAs, GaAs, and AlAs.

${ }^{26}$ Z. H. Lin, T. Y. Wang, G. B. Stringfellow, and P. C. Taylor, Appl. Phys. Lett. 52, 1590 (1988).

${ }^{27}$ K. J. Moore, G. Duggan, K. Woodbridge, C. Roberts, N. Pulsford, and R. J. Nicholas, Phys. Rev. B 42, 3024 (1990). 